\title{
Form factor decomposition of the off-shell four-gluon amplitudes
}

\author{
Naser Ahmadiniaz* and Christian Schubert \\ Instituto de Física y Matemáticas \\ Universidad Michoacana de San Nicolás de Hidalgo \\ Edificio C-3, Apdo. Postal 2-82 \\ C.P. 58040, Morelia, Michoacán, México \\ E-mail: nasereifm.umich.mx, schuberteifm.umich.mx
}

\begin{abstract}
We show how to use the Bern-Kosower master formula, originally a generating functional for on-shell gluon matrix elements, to derive well-organized form factor decompositions of the offshell one-particle-irreducible $\mathrm{N}$ - gluon vertices. Two such algorithms are presented which can be used for any $\mathrm{N}$, the first one optimized with respect to the nonabelian gauge invariance, the second one with respect to transversality. We give explicit results for the three- and four-gluon cases. The second algorithm in the three-point case reproduces precisely the well-known BallChiu decomposition, and in the four-point case a natural generalization thereof. A particularly simple structure emerges in the $\mathscr{N}=4$ SYM case.
\end{abstract}

QCD-TNT-III-From quarks and gluons to hadronic matter: A bridge too far?,

2-6 September, 2013

European Centre for Theoretical Studies in Nuclear Physics and Related Areas (ECT*), Villazzano, Trento (Italy)

${ }^{*}$ Speaker. 


\section{Introduction}

Multi-gluon amplitudes in perturbative QCD pose two very different computational challenges. On one hand, there is the calculation of on-shell matrix elements, a field that has seen tremendous activity and progress during the last decade, particularly so for the massless and/or SUSY cases. A whole host of new concepts and techniques have emerged, such as unitarity-based methods [1, 2], twistors [3], BCFW recursion [4, 5], and Grassmannians [6, 7]; see [8] and [9] for recent reviews.

On the other hand, there are the off-shell gluon amplitudes, which carry additional physical information, particularly on the infrared properties of QCD (see, e.g., [10]). They are also essential for the matching of perturbative information with lattice data (see, e.g., [11]), and constitute an essential ingredient for the Schwinger-Dyson equations. In the latter context, previously only the two and three-point amplitudes were used with their full loop-corrected structure, but a need for the inclusion of the four-gluon vertex is already felt [12]. Off-shell, the natural building blocks are not the connected but the one-particle-irreducible ('1PI') $N$ - gluon Green's functions, also called ' $N$ - gluon vertices'. For their calculation, no progress comparable to the on-shell case was achieved in recent years, and their explicit computation is presently essentially still stuck at the three-point level.

Let us shortly review what is known about the $N$ - gluon vertices. Following early work by Celmaster and Gonsalves [13] and Pascual and Tarrach [14], in 1980 Ball and Chiu [15] studied the off-shell gluon amplitudes for the gluon loop in Feynman gauge. They analyzed the Ward identities and derived a form factor ("Ball-Chiu") decomposition of the three-gluon vertex, valid to all loop orders. Cornwall and Papavassiliou [16] in 1989 constructed a "gauge invariant three-gluon vertex" through the pinch technique (see [17] for a review of this technique). Freedman et al. in 1992 studied the conformal properties of this vertex [18]. In 1993 J. Papavassiliou [19] extended this construction to the four-gluon vertex. Davydychev, Osland and Tarasov [20] in 1996 calculated the gluon loop contribution to the one-loop three-gluon vertex in arbitrary covariant gauge, and also the massless fermion loop contribution. The fermion loop calculation was later generalized to the massive case by Davydychev, Osland and Saks [21]. In 2006, Binger and Brodsky [22] studied the one-loop three-gluon vertex in various dimensions, using the background field method $[23,24]$. Besides the gluon and fermion loop cases, they now also included the scalar loop, as is needed for SUSY extensions of QCD, and they derived various sum rules relevant to the SUSY case. They also verified that, as had been suggested in [25, 26], in the gluon loop case the use of the background field method with quantum Feynman gauge leads to precisely the same vertex as the pinch technique. At two loops, the three-gluon vertex has been obtained so far only for some very special momentum configurations $[27,28,29])$.

Our aim here is to demonstrate that the string-inspired approach to perturbative QCD, originally developed in the on-shell context by Bern and Kosower [30, 31, 32], is also extremely promising as a tool for the derivation of form factor decompositions of the $N$ - gluon amplitudes off-shell (see also J. Cornwall's talk at this workshop [33]). 


\section{The Ward identities for the off-shell gluon amplitudes}

Off-shell, the Ward identities for the gluon amplitudes are inhomogeneous and map the $N$ vertex to $N-1$ - point amplitudes. E.g. for the four-point case one finds [19]

$$
p_{1}^{\mu} \Gamma_{\mu v \alpha \beta}^{a b c d}\left(p_{1}, p_{2}, p_{3}, p_{4}\right)=f_{a b e} \Gamma_{v \alpha \beta}^{c d e}\left(p_{1}+p_{2}, p_{3}, p_{4}\right)+\text { perm. }
$$

These identities as they stand hold for the scalar and spinor loop unambiguously, but for the gluon loop only if one uses the pinch technique, or equivalently the background field method with quantum Feynman gauge. Other gauge fixings of the gluon loop will generally lead to a more complicated right-hand side involving ghosts.

\section{The QCD three-gluon vertex and its Ball-Chiu decomposition}

The three-gluon vertex in QCD at tree level,

$$
-i g f^{a_{1} a_{2} a_{3}}\left[g_{\mu_{1} \mu_{2}}\left(p_{1}-p_{2}\right) \mu_{3}+c y c l .\right]
$$

is corrected at the one-loop level by the 1PI three-gluon vertex with a spinor or gluon loop. E.g. for the spinor loop case we have the diagram shown in Fig. 1 (and a second one with the other orientation of the fermion).

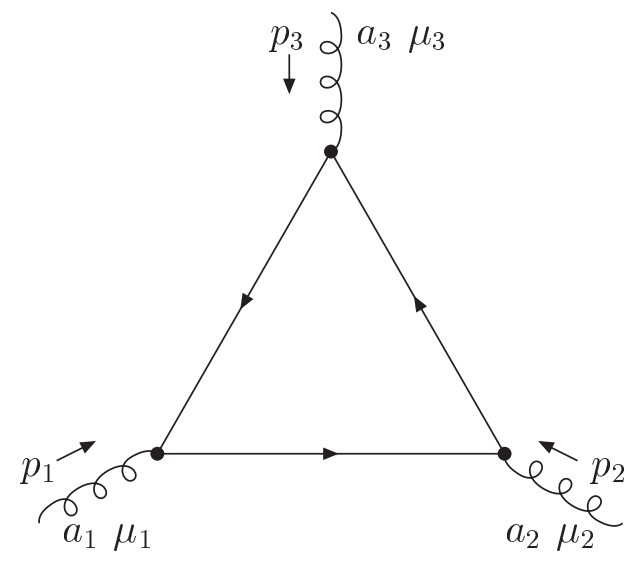

Figure 1: Three-gluon vertex.

The Ball-Chiu decomposition of the vertex is [15]

$$
\begin{aligned}
\Gamma_{\mu_{1} \mu_{2} \mu_{3}}\left(p_{1}, p_{2}, p_{3}\right) & =A\left(p_{1}^{2}, p_{2}^{2} ; p_{3}^{2}\right) g_{\mu_{1} \mu_{2}}\left(p_{1}-p_{2}\right)_{\mu_{3}}+B\left(p_{1}^{2}, p_{2}^{2} ; p_{3}^{2}\right) g_{\mu_{1} \mu_{2}}\left(p_{1}+p_{2}\right)_{\mu_{3}} \\
+ & C\left(p_{1}^{2}, p_{2}^{2} ; p_{3}^{2}\right)\left[p_{1 \mu_{2}} p_{2 \mu_{1}}-p_{1} \cdot p_{2} g_{\mu_{1} \mu_{2}}\right]\left(p_{1}-p_{2}\right)_{\mu_{3}} \\
+ & \frac{1}{3} S\left(p_{1}^{2}, p_{2}^{2}, p_{3}^{2}\right)\left(p_{1 \mu_{3}} p_{2 \mu_{1}} p_{3 \mu_{2}}+p_{1 \mu_{2}} p_{2 \mu_{3}} p_{3 \mu_{1}}\right) \\
+ & F\left(p_{1}^{2}, p_{2}^{2} ; p_{3}^{2}\right)\left[p_{1 \mu_{2}} p_{2 \mu_{1}}-p_{1} \cdot p_{2} g_{\mu_{1} \mu_{2}}\right]\left[p_{2 \mu_{3}} p_{1} \cdot p_{3}-p_{1 \mu_{3}} p_{2} \cdot p_{3}\right]
\end{aligned}
$$




$$
\begin{aligned}
& +H\left(p_{1}^{2}, p_{2}^{2}, p_{3}^{2}\right)\left(-g_{\mu_{1} \mu_{2}}\left[p_{1 \mu_{3}} p_{2} \cdot p_{3}-p_{2 \mu_{3}} p_{1} \cdot p_{3}\right]+\frac{1}{3}\left(p_{1 \mu_{3}} p_{2 \mu_{1}} p_{3 \mu_{2}}-p_{1 \mu_{2}} p_{2 \mu_{3}} p_{3 \mu_{1}}\right)\right) \\
& +\left[\text { cyclic permutations of }\left(p_{1}, \mu_{1}\right),\left(p_{2}, \mu_{2}\right),\left(p_{3}, \mu_{3}\right)\right] .
\end{aligned}
$$

This form factor decomposition is universal, that is, valid for the scalar, spinor and gluon loop, and also for higher loop corrections. Only the coefficient functions $A, B, C, F, H, S$ change. At tree level, $A=1$, the other functions vanish. Explicit calculation shows that $S$ still vanishes at one-loop. The tensor structures multiplying $F, H$ are manifestly transversal.

\section{The string-inspired formalism}

In 1991 Bern and Kosower in their seminal work derived, by an analysis of the infinite string limit of certain string amplitudes, the following Bern-Kosower master formula [30, 31, 32]:

$$
\begin{aligned}
\Gamma^{a_{1} \ldots a_{N}}\left[p_{1}, \varepsilon_{1} ; \ldots ; p_{N}, \varepsilon_{N}\right]= & (-i g)^{N} \operatorname{tr}\left(T^{a_{1}} \ldots T^{a_{N}}\right) \int_{0}^{\infty} d T(4 \pi T)^{-D / 2} e^{-m^{2} T} \\
& \times \int_{0}^{T} d \tau_{1} \int_{0}^{\tau_{1}} d \tau_{2} \ldots \int_{0}^{\tau_{N-2}} d \tau_{N-1} \\
& \times\left.\exp \left\{\sum_{i, j=1}^{N}\left[\frac{1}{2} G_{B i j} p_{i} \cdot p_{j}-i \dot{G}_{B i j} \varepsilon_{i} \cdot p_{j}+\frac{1}{2} \ddot{G}_{B i j} \varepsilon_{i} \cdot \varepsilon_{j}\right]\right\}\right|_{\operatorname{lin}\left(\varepsilon_{1} \ldots \varepsilon_{\mathrm{N}}\right)}
\end{aligned}
$$

As it stands, this is a parameter integral representation for the (color-ordered) 1PI $N$ - gluon amplitude induced by a scalar loop, with momenta $p_{i}$ and polarizations $\varepsilon_{i}$, in $D$ dimensions. Here $m$ and $T$ are the loop mass and proper-time, $\tau_{i}$ fixes the location of the $i$ th gluon, and $G_{B i j} \equiv G_{B}\left(\tau_{i}, \tau_{j}\right)$ denotes the "bosonic" worldline Green's function, defined by

$$
G_{B i j}=\left|\tau_{i}-\tau_{j}\right|-\frac{\left(\tau_{i}-\tau_{j}\right)^{2}}{T}
$$

and dots generally denote a derivative acting on the first variable. Explicitly,

$$
\begin{aligned}
& \dot{G}_{B}\left(\tau_{1}, \tau_{2}\right)=\operatorname{sign}\left(\tau_{1}-\tau_{2}\right)-2 \frac{\left(\tau_{1}-\tau_{2}\right)}{T} \\
& \ddot{G}_{B}\left(\tau_{1}, \tau_{2}\right)=2 \delta\left(\tau_{1}-\tau_{2}\right)-\frac{2}{T}
\end{aligned}
$$

In the Bern-Kosower formalism, the master formula serves as a generating functional for the full on-shell $N$ - gluon amplitudes for the scalar, spinor and gluon loop, through the use of the BernKosower rules:

- For fixed $N$, expand the generating exponential and take only the terms linear in all polarization vectors. 
- Use suitable integrations-by-parts (IBPs) to remove all second derivatives $\ddot{G}_{B i j}$.

- Apply two types of pattern-matching rules:

- The "tree replacement rules" generate (from a field theory point of view) the contributions of the missing reducible diagrams.

- The "loop replacement rules" generate the integrands for the spinor and gluon loop from the one for the scalar loop.

\section{The worldline path integral approach}

Shortly after the work of Bern and Kosower, Strassler [34] rederived the master formula and the loop replacement rules using worldline path integral representations of the gluonic effective actions. E.g. for the scalar loop,

$$
\Gamma[A]=\operatorname{tr} \int_{0}^{\infty} \frac{d T}{T} e^{-m^{2} T} \int \mathscr{D} x(\tau) \mathscr{P} e^{-\int_{0}^{T} d \tau\left(\frac{1}{4} \dot{x}^{2}+i g \dot{x} \cdot A(x(\tau))\right)},
$$

where $A_{\mu}=A_{\mu}^{a} T^{a}$ and $\mathscr{P}$ denotes path ordering. This also showed that the master formula and the loop replacement rules hold off-shell, which was not obvious from its original derivation. Moreover, in a beautiful but unpublished paper [35] Strassler noted that the IBP generates automatically abelian field strength tensors

$$
f_{i}^{\mu v} \equiv p_{i}^{\mu} \varepsilon_{i}^{v}-\varepsilon_{i}^{\mu} p_{i}^{v}
$$

in the bulk, and color commutators $\left[T^{a_{i}}, T^{a_{j}}\right]$ as boundary terms. Those fit together to produce full nonabelian field strength tensors

$$
F_{\mu \nu} \equiv F_{\mu \nu}^{a} T^{a}=\left(\partial_{\mu} A_{v}^{a}-\partial_{\nu} A_{\mu}^{a}\right) T^{a}+i g\left[A_{\mu}^{b} T^{b}, A_{v}^{c} T^{c}\right],
$$

in the low-energy effective action. Thus we see something very interesting, namely the emergence of gauge invariant tensor structures at the integrand level.

However, the removal of all $\ddot{G}_{B i j}$ by IBP can be done in many ways! Moreover, it is not obvious how to do it without breaking the bose symmetry between the gluons. In [35] Strassler started to investigate this ambiguity at the four-point level, but an algorithm valid for any $N$ and manifestly preserving the permutation invariance was given only much later $[36,37]$ by one of the authors. This algorithm still followed the objective of achieving a form of the $N$ - gluon vertex that, in $x$ - space, would correspond to a manifestly covariant representation of the nonabelian effective action. However, it turns out not be optimized from another point of view, which is important, e.g., for the Schwinger-Dyson equations, namely it does not lead to a clean separation of the vertices into transversal and longitudinal parts. This remaining obstacle has been overcome only recently in [38], where we give two IBP algorithms that work for arbitrary $N$ and lead to explicit form-factor decompositions of the off-shell $N$ - gluon amplitudes: 
- The first algorithm uses only local total derivative terms and leads to a representation that matches term-by-term with the low-energy effective action ("Q-representation ”).

- The second algorithm uses both local and nonlocal total derivative terms and leads to the transversality of all bulk terms at the integrand level ("S-representation").

In [39], we applied both algorithms to the three-point case and showed that, in particular, the second algorithm generates precisely the Ball-Chiu decomposition. Very recently, we have carried out the same program also for the four-gluon vertex [40]. We will now sketch these rather involved calculations as well as space permits.

\section{The Q-representation of the three-gluon vertex}

For $N=3$, the Q-representation is (for the scalar loop) [39]

$$
\Gamma=\frac{g^{3}}{(4 \pi)^{\frac{D}{2}}} \operatorname{tr}\left(T^{a_{1}}\left[T^{a_{2}}, T^{a_{3}}\right]\right)\left(\Gamma^{3}+\Gamma^{2}+\Gamma^{\mathrm{bt}}\right),
$$

where

$$
\begin{aligned}
\Gamma^{3} & =-\int_{0}^{\infty} \frac{d T}{T^{\frac{D}{2}}} e^{-m^{2} T} \int_{0}^{T} d \tau_{1} \int_{0}^{\tau_{1}} d \tau_{2} Q_{3}^{3} \exp \left\{\sum_{i, j=1}^{3} \frac{1}{2} G_{B i j} p_{i} \cdot p_{j}\right\} \\
\Gamma^{2} & =\Gamma^{3}\left(Q_{3}^{3} \rightarrow Q_{3}^{2}\right) \\
\Gamma^{\mathrm{bt}} & =\int_{0}^{\infty} \frac{d T}{T^{\frac{D}{2}}} e^{-m^{2} T} \int_{0}^{T} d \tau_{1} \dot{G}_{B 12} \dot{G}_{B 21}\left[\varepsilon_{3} \cdot f_{1} \cdot \varepsilon_{2} e^{G_{B 12} p_{1} \cdot\left(p_{2}+p_{3}\right)}+\text { cycl. }\right],
\end{aligned}
$$

and

$$
\begin{aligned}
Q_{3}^{3} & =\dot{G}_{B 12} \dot{G}_{B 23} \dot{G}_{B 31} \operatorname{tr}\left(f_{1} f_{2} f_{3}\right) \\
Q_{3}^{2} & =\frac{1}{2} \dot{G}_{B 12} \dot{G}_{B 21} \operatorname{tr}\left(f_{1} f_{2}\right) \dot{G}_{B 3 k} \varepsilon_{3} \cdot p_{k}+2 \text { perm }
\end{aligned}
$$

Here $\Gamma^{\mathrm{bt}}$ comes from the boundary terms, and the upper indices on $\Gamma^{2,3}, Q^{2,3}$ refer to the "cycle content"; e.g. $Q_{3}^{3}$ contains a factor $\dot{G}_{B 12} \dot{G}_{B 23} \dot{G}_{B 31}$ whose indices form a closed cycle involving three points, called "three-cycle". Dummy indices like the one appearing in $Q_{3}^{2}$ are to be summed from 1 to $N=3$. To pass from the scalar to the spinor loop, one applies the "loop replacement rules"

$$
\begin{aligned}
\dot{G}_{B i j} \dot{G}_{B j i} & \rightarrow \dot{G}_{B i j} \dot{G}_{B j i}-G_{F i j} G_{F j i} \\
\dot{G}_{B 12} \dot{G}_{B 23} \dot{G}_{B 31} & \rightarrow \dot{G}_{B 12} \dot{G}_{B 23} \dot{G}_{B 31}-G_{F 12} G_{F 23} G_{F 31},
\end{aligned}
$$


where $G_{F i j}=\operatorname{sign}\left(\tau_{i}-\tau_{j}\right)$. Similarly, the integrand for the gluon loop is obtained from the scalar loop one by

$$
\begin{aligned}
\dot{G}_{B i j} \dot{G}_{B j i} & \rightarrow \dot{G}_{B i j} \dot{G}_{B j i}-4 G_{F i j} G_{F j i} \\
\dot{G}_{B 12} \dot{G}_{B 23} \dot{G}_{B 31} & \rightarrow \dot{G}_{B 12} \dot{G}_{B 23} \dot{G}_{B 31}-4 G_{F 12} G_{F 23} G_{F 31} .
\end{aligned}
$$

As stated above, the gluon loop vertex obtained in this way corresponds to the background field method with quantum Feynman gauge $[34,41]$. And for all three cases - scalar, spinor and gluon loop - the vertex allows a perfect match with the low-energy effective action. We recall that the low energy expansion of the one-loop QCD effective action induced by a loop particle of mass $m$ has the form (see, e.g., [42])

$$
\Gamma[F]=\int_{0}^{\infty} \frac{d T}{T} \frac{\mathrm{e}^{-m^{2} T}}{(4 \pi T)^{D / 2}} \operatorname{tr} \int d x_{0} \sum_{n=2}^{\infty} \frac{(-T)^{n}}{n !} O_{n}[F],
$$

where $O_{n}(F)$ is a Lorentz and gauge invariant expression of mass dimension $2 n$. To lowest orders,

$$
\begin{aligned}
& O_{2}=c_{2} g^{2} F_{\mu v} F_{\mu v}, \\
& O_{3}=c_{3}^{3} i g^{3} F_{\kappa \lambda} F_{\lambda \mu} F_{\mu \kappa}+c_{3}^{2} g^{2} D_{\lambda} F_{\mu v} D^{\lambda} F^{\mu v},
\end{aligned}
$$

where only the coefficients $c_{2}, c_{3}^{2,3}$ depend on the spin of the loop particle. We recognize the correspondences

$$
\begin{aligned}
& \Gamma^{3} \leftrightarrow F_{\kappa}{ }^{\lambda} F_{\lambda}{ }^{\mu} F_{\mu}{ }^{\kappa}=f_{\kappa}^{\lambda} f_{\lambda}^{\mu} f_{\mu}^{\kappa}+\text { higher point terms } \\
& \Gamma^{2} \leftrightarrow(\partial+i g \underbrace{A) F(\partial}+i g A) F \\
& \Gamma^{\mathrm{bt}} \leftrightarrow(f+i g \underbrace{[A, A])(f}+i g[A, A]) .
\end{aligned}
$$

\section{The S-representation of the three-gluon vertex}

In the S-representation, the three-gluon vertex becomes

$$
\tilde{\Gamma}=\frac{g^{3}}{(4 \pi)^{\frac{D}{2}}} \operatorname{tr}\left(T^{a_{1}}\left[T^{a_{2}}, T^{a_{3}}\right]\right)\left(\tilde{\Gamma}^{3}+\tilde{\Gamma}^{2}+\tilde{\Gamma}^{\mathrm{bt}}\right)
$$

where 


$$
\begin{aligned}
& \tilde{\Gamma}^{3}=-\int_{0}^{\infty} \frac{d T}{T^{\frac{D}{2}}} e^{-m^{2} T} \int_{0}^{T} d \tau_{1} \int_{0}^{\tau_{1}} d \tau_{2} S_{3}^{3} \exp \left\{\sum_{i, j=1}^{3} \frac{1}{2} G_{B i j} p_{i} \cdot p_{j}\right\} \\
& \tilde{\Gamma}^{2}=\tilde{\Gamma}^{3}\left(S_{3}^{3} \rightarrow S_{3}^{2}\right) \\
& \tilde{\Gamma}^{\mathrm{bt}}=\int_{0}^{\infty} \frac{d T}{T^{\frac{D}{2}}} e^{-m^{2} T} \int_{0}^{T} d \tau_{1} \dot{G}_{B 12} \dot{G}_{B 21}\left\{\left[\varepsilon_{3} \cdot f_{1} \cdot \varepsilon_{2}-\frac{1}{2} \operatorname{tr}\left(f_{1} f_{2}\right) \rho_{3}+\frac{1}{2} \operatorname{tr}\left(f_{3} f_{1}\right) \rho_{2}\right]\right. \\
& \left.\times e^{G_{B 12} p_{1} \cdot\left(p_{2}+p_{3}\right)}+\mathrm{cycl} .\right\} \text {, }
\end{aligned}
$$

and

$$
\begin{aligned}
& S_{3}^{3}=\dot{G}_{B 12} \dot{G}_{B 23} \dot{G}_{B 31} \operatorname{tr}\left(f_{1} f_{2} f_{3}\right) \\
& S_{3}^{2}=\frac{1}{2} \dot{G}_{B 12} \dot{G}_{B 21} \operatorname{tr}\left(f_{1} f_{2}\right) \dot{G}_{B 3 k} \frac{r_{3} \cdot f_{3} \cdot p_{k}}{r_{3} \cdot p_{3}}+2 \text { perm. }
\end{aligned}
$$

Here we have introduced three vectors $r_{i}$ which obey $r_{i} \cdot p_{i} \neq 0$ but are arbitrary otherwise, and $\rho_{i}:=\frac{r_{i} \cdot \varepsilon_{i}}{r_{i} \cdot p_{i}}$. Note that $S^{3}$ is the same as $Q^{3}$ above, but that in $S^{2}$, contrary to $Q^{2}$, all three polarization vectors $\varepsilon_{i}$ are absorbed in abelian field strength tensors $f_{i}$. Thus all bulk terms are now manifestly transversal, even at the integrand level, and it turns out that with the cyclic choice

$$
r_{1}=p_{2}-p_{3}, r_{2}=p_{3}-p_{1}, r_{3}=p_{1}-p_{2},
$$

we get a term-by-term match with the Ball-Chiu decomposition:

$$
\begin{aligned}
& H\left(p_{1}^{2}, p_{2}^{2}, p_{3}^{2}\right)=C(r) \frac{d_{0} g^{2}}{(4 \pi)^{D / 2}} \Gamma\left(3-\frac{D}{2}\right) I_{3, B}^{D}\left(p_{1}^{2}, p_{2}^{2}, p_{3}^{2}\right) \\
& A\left(p_{1}^{2}, p_{2}^{2} ; p_{3}^{2}\right)=C(r) \frac{d_{0} g^{2}}{2(4 \pi)^{D / 2}} \Gamma\left(2-\frac{D}{2}\right)\left[I_{\mathrm{bt}, \mathrm{B}}^{D}\left(p_{1}^{2}\right)+I_{\mathrm{bt}, \mathrm{B}}^{D}\left(p_{2}^{2}\right)\right] \\
& B\left(p_{1}^{2}, p_{2}^{2} ; p_{3}^{2}\right)=C(r) \frac{d_{0} g^{2}}{2(4 \pi)^{D / 2}} \Gamma\left(2-\frac{D}{2}\right)\left[I_{\mathrm{bt}, \mathrm{B}}^{D}\left(p_{1}^{2}\right)-I_{\mathrm{bt}, \mathrm{B}}^{D}\left(p_{2}^{2}\right)\right] \\
& F\left(p_{1}^{2}, p_{2}^{2} ; p_{3}^{2}\right)=C(r) \frac{d_{0} g^{2}}{(4 \pi)^{D / 2}} \Gamma\left(3-\frac{D}{2}\right) \frac{I_{2, B}^{D}\left(p_{1}^{2}, p_{2}^{2}, p_{3}^{2}\right)-I_{2, B}^{D}\left(p_{2}^{2}, p_{1}^{2}, p_{3}^{2}\right)}{p_{1}^{2}-p_{2}^{2}} \\
& C\left(p_{1}^{2}, p_{2}^{2} ; p_{3}^{2}\right)=C(r) \frac{d_{0} g^{2}}{(4 \pi)^{D / 2}} \Gamma\left(2-\frac{D}{2}\right) \frac{I_{\mathrm{bt}, \mathrm{B}}^{D}\left(p_{1}^{2}\right)-I_{\mathrm{bt}, \mathrm{B}}^{D}\left(p_{2}^{2}\right)}{p_{1}^{2}-p_{2}^{2}} \\
& S\left(p_{1}^{2}, p_{2}^{2} ; p_{3}^{2}\right)=0,
\end{aligned}
$$

where we have used $\operatorname{tr}\left(T^{a_{1}}\left[T^{a_{2}}, T^{a_{3}}\right]\right)=i C(r) f^{a_{1} a_{2} a_{3}}$.

Here we have written down the scalar loop case, but due to the loop replacement rules (6.4) and (6.5), the spinor and gluon loop cases differ from it only in the coefficient functions on the righthand sides. For completeness, let us write down these coefficient functions also in terms of standard Feynman-Schwinger parameter integrals (again for the scalar loop) 


$$
\begin{aligned}
I_{3, B}^{D}\left(p_{1}^{2}, p_{2}^{2}, p_{3}^{2}\right) & =\int_{0}^{1} d \alpha_{1} d \alpha_{2} d \alpha_{3} \delta\left(1-\alpha_{1}-\alpha_{2}-\alpha_{3}\right) \times \frac{\left(1-2 \alpha_{1}\right)\left(1-2 \alpha_{2}\right)\left(1-2 \alpha_{3}\right)}{\left(m^{2}+\alpha_{1} \alpha_{2} p_{1}^{2}+\alpha_{2} \alpha_{3} p_{2}^{2}+\alpha_{1} \alpha_{3} p_{3}^{2}\right)^{3-\frac{D}{2}}} \\
I_{2, B}^{D}\left(p_{1}^{2}, p_{2}^{2}, p_{3}^{2}\right) & =\int_{0}^{1} d \alpha_{1} d \alpha_{2} d \alpha_{3} \delta\left(1-\alpha_{1}-\alpha_{2}-\alpha_{3}\right) \times \frac{\left(1-2 \alpha_{2}\right)^{2}\left(1-2 \alpha_{1}\right)}{\left(m^{2}+\alpha_{1} \alpha_{2} p_{1}^{2}+\alpha_{2} \alpha_{3} p_{2}^{2}+\alpha_{1} \alpha_{3} p_{3}^{2}\right)^{3-\frac{D}{2}}} \\
I_{b t, B}^{D}\left(p^{2}\right) & =\int_{0}^{1} d \alpha \frac{(1-2 \alpha)^{2}}{\left(m^{2}+\alpha(1-\alpha) p^{2}\right)^{2-\frac{D}{2}}} .
\end{aligned}
$$

They are, of course, of the same type as the ones arising in other approaches.

\section{The four-gluon vertex}

Proceeding to the four-point case, here the Q-representation for the scalar loop has the following bulk terms:

$$
\begin{aligned}
\Gamma_{1}^{a_{1} a_{2} a_{3} a_{4}}= & g^{4} \operatorname{tr}\left(T^{a_{1}} \ldots T^{a_{4}}\right) \int_{0}^{\infty} d T(4 \pi T)^{-D / 2} e^{-m^{2} T} \\
& \quad \times \int_{0}^{T} d \tau_{1} \int_{0}^{\tau_{1}} d \tau_{2} \int_{0}^{\tau_{2}} d \tau_{3} Q_{4} \exp \left\{\sum_{i, j=1}^{4} \frac{1}{2} G_{B i j} p_{i} \cdot p_{j}\right\}, \\
Q_{4}= & Q_{4}^{4}+Q_{4}^{3}+Q_{4}^{2}-Q_{4}^{22} \\
Q_{4}^{4}= & \dot{G}(1234)+\dot{G}(1243)+\dot{G}(1324) \\
Q_{4}^{3}= & \dot{G}(123) T(4)+\dot{G}(234) T(1)+\dot{G}(341) T(2)+\dot{G}(412) T(3) \\
Q_{4}^{2}= & \dot{G}(12) T(34)+\dot{G}(13) T(24)+\dot{G}(14) T(23)+\dot{G}(23) T(14) \\
& +\dot{G}(24) T(13)+\dot{G}(34) T(12) \\
Q_{4}^{22}= & \dot{G}(12) \dot{G}(34)+\dot{G}(13) \dot{G}(24)+\dot{G}(14) \dot{G}(23),
\end{aligned}
$$

where we have now employed a more condensed notation:

$$
\begin{aligned}
\dot{G}\left(i_{1} i_{2} \cdots i_{n}\right) & :=\dot{G}_{B i_{1} i_{2}} \dot{G}_{B i_{2} i_{3}} \cdots \dot{G}_{B i_{n} i_{1}}\left(\frac{1}{2}\right)^{\delta_{n, 2}} \operatorname{tr}\left(f_{i_{1}} f_{i_{2}} \cdots f_{i_{n}}\right) \\
T(i) & :=\sum_{r} \dot{G}_{B i r} \varepsilon_{i} \cdot p_{r} \\
T(i j) & :=\sum_{r, s}\left\{\dot{G}_{B i r} \varepsilon_{i} \cdot p_{r} \dot{G}_{j s} \varepsilon_{j} \cdot p_{s}+\frac{1}{2} \dot{G}_{B i j} \varepsilon_{i} \cdot \varepsilon_{j}\left[\dot{G}_{B i r} p_{i} \cdot p_{r}-\dot{G}_{B j r} p_{j} \cdot p_{r}\right]\right\} .
\end{aligned}
$$


The IBP procedure now leads to both single boundary terms (three-point integrals) and double boundary terms (two-point integrals). The following rules emerge:

- Each single boundary term, say for the limit $3 \rightarrow 4$, matches some bulk term in the Qrepresentation of the three-gluon vertex, with momenta $\left(p_{1}, p_{2}, p_{3}+p_{4}\right)$, and $f_{3}=p_{3} \otimes \varepsilon_{3}-$ $\varepsilon_{3} \otimes p_{3}$ replaced by $\varepsilon_{3} \otimes \varepsilon_{4}-\varepsilon_{4} \otimes \varepsilon_{3}$.

- Each double boundary term, say for the limit $1 \rightarrow 2,3 \rightarrow 4$, matches the bulk term in the Q-representation of the two-point function, with momenta $\left(p_{1}+p_{2}, p_{3}+p_{4}\right)$, and the double replacement

$$
\begin{aligned}
& f_{1}=p_{1} \otimes \varepsilon_{1}-\varepsilon_{1} \otimes p_{1} \rightarrow \varepsilon_{1} \otimes \varepsilon_{2}-\varepsilon_{2} \otimes \varepsilon_{1} \\
& f_{2}=p_{2} \otimes \varepsilon_{2}-\varepsilon_{2} \otimes p_{2} \rightarrow \varepsilon_{3} \otimes \varepsilon_{4}-\varepsilon_{4} \otimes \varepsilon_{3}
\end{aligned}
$$

Effectively, a boundary term always completes a $f_{i}$ to a full nonabelian field strength tensor; that is, we are seeing just the projection to plane waves of the completion

$$
\partial_{\mu} A_{v}-\partial_{v} A_{\mu} \rightarrow \partial_{\mu} A_{v}-\partial_{v} A_{\mu}+i g\left[A_{\mu}, A_{v}\right]
$$

Moreover, this recursive structure is compatible with the replacement rules.

The S-representation looks similar, but has the bulk terms written completely in terms of the $f_{i}$, so that all non-transversality has now been pushed into the boundary terms. It involves now the choice of four vectors $r_{i}$ with $r_{i} \cdot p_{i} \neq 0$.

\section{Off-shell one-loop four-gluon vertex in $\mathscr{N}=4 \mathrm{SYM}$}

In $\mathscr{N}=4 \mathrm{SYM}$ the one-loop two - and three - gluon amplitudes vanish (this relates to the conformal invariance and finiteness of the theory). The one-loop four-gluon vertex is the first non-vanishing one, and it is extremely simple: all boundary terms cancel out (since they would covariantize the nonexisting lower point amplitudes) and the bulk term factors as

$$
\Gamma^{a_{1} a_{2} a_{3} a_{4}}=4 g^{4} \operatorname{tr}\left(T^{a_{1}} T^{a_{2}} T^{a_{3}} T^{a_{4}}\right) F_{\mathrm{ss}}^{4} B(1234)+\text { non }- \text { cyclic permutations },
$$

where $B(1234)$ is the off-shell scalar box integral with momenta $p_{1}, \ldots, p_{4}$, and the whole Lorentz structure is contained in the invariant

$$
\begin{aligned}
F_{\mathrm{ss}}^{4}= & \operatorname{tr}\left(f_{1} f_{2} f_{3} f_{4}\right)+\operatorname{tr}\left(f_{1} f_{2} f_{4} f_{3}\right)+\operatorname{tr}\left(f_{1} f_{3} f_{2} f_{4}\right) \\
& -\frac{1}{4} \operatorname{tr}\left(f_{1} f_{2}\right) \operatorname{tr}\left(f_{3} f_{4}\right)-\frac{1}{4} \operatorname{tr}\left(f_{1} f_{3}\right) \operatorname{tr}\left(f_{2} f_{4}\right)-\frac{1}{4} \operatorname{tr}\left(f_{1} f_{4}\right) \operatorname{tr}\left(f_{2} f_{3}\right) .
\end{aligned}
$$


Although this explicit form of the off-shell one-loop four-gluon amplitude in $\mathscr{N}=4$ SYM appears to be new, the Lorentz tensor $F_{\mathrm{ss}}$ is well-known to string theorists, since it appears in the low energy expansion of the effective action of the open superstring; see [43] and refs therein.

\section{Summary and Outlook}

To summarize, the main points which we wanted to make here are:

- In the string-inspired formalism, form factor decompositions of the $N$ - vertex compatible with Bose symmetry and gauge invariance can be generated simply by an integration-byparts procedure starting from the Bern-Kosower master formalism, which originally was derived as a generating functional for on-shell matrix elements.

- At the one-loop level, the parameter integrals appearing in the form factors for the scalar, spinor and gluon loop cases are all obtained directly from the Bern-Kosower master formula.

- We have carried out this program explicitly for the three- and four-point cases.

- In particular, we have obtained a natural four-point generalization of the Ball-Chiu decomposition. It is distinguished by the fact that all true four-point terms are manifestly transversal, so that all longitudinal components are given by lower-point integrals.

\section{References}

[1] Z. Bern, L. J. Dixon, D. C. Dunbar and D. A. Kosower, Nucl. Phys. B 425 (1994) 217, hep-ph/9403226.

[2] Z. Bern and Y.-t. Huang, J. Phys. A 44 (2011) 454003, arXiv:1103.1869 [hep-th].

[3] E. Witten, Comm. Math. Phys. 252 (2004) 189, hep-th/0312171.

[4] R. Britto, F. Cachazo and B. Feng, Nucl. Phys. B 715 (2005), 499, hep-th/0412308.

[5] R. Britto, F. Cachazo, B. Feng and E. Witten, Phys. Rev. Lett. 94 (2005) 181602, hep-th/0501052.

[6] N. Arkani-Hamed, F. Cachazo, C. Cheung and J. Kaplan, JHEP 1003 (2010) 020, arXiv:0907.5418 [hep-th].

[7] L. J. Mason and D. Skinner, JHEP 0911 (2009) 045, arXiv:0909.0250 [hep-th].

[8] H. Elvang and Y.-t. Huang, arXiv:1308.1697 [hep-th].

[9] L. J. Dixon, SLAC-PUB-15775, arXiv:1310.5353 [hep-ph].

[10] R. Alkofer, M. Q. Huber and K. Schwenzer, Eur. Phys. J. C 62 (2009) 761, arXiv:0812.4045 [hep-ph].

[11] M. Pelaez, M. Tissier and N. Wschebor, arXiv:1310.2594 [hep-th].

[12] C. Kellermann and C. Fischer, Phys. Rev. D 78 (2008) 025015, arXiv: 0801.2697 [hep-th]; V. Mader and R. Alkofer, PoS ConfinementX (2012) 063, arXiv:1301.7498 [hep-th] .

[13] W. Celmaster and R. J. Gonsalves, Phys. Rev. D 20 (1979) 1420.

[14] P. Pascual and R. Tarrach, Nucl. Phys. B 174 (1980) 123, Erratum-ibid. B 181 (1981) 546. 
[15] J. S. Ball and T. W. Chiu, Phys. Rev. D 22 (1980) 2250, Erratum ibido 23 (1981) 3085.

[16] J. M. Cornwall and J. Papavassiliou, Phys. Rev. D 40 (1989) 3474.

[17] D. Binosi and J. Papavassiliou, Phys. Rept. 479 (2009) 1, arXiv:0909.2536 [hep-ph].

[18] D. Z. Freedman, G. Grignani, K. Johnson and N. Rius, Ann. Phys. (N. Y.) 218 (1992) 75.

[19] J. Papavassiliou, Phys. Rev. D 47 (1993) 4728.

[20] A. I. Davydychev, P. Osland and O. V. Tarasov, Phys. Rev. D 54(1996) 4087, hep-ph/9605348; Erratum-ibido 59 (1999) 109901.

[21] A. I. Davydychev, P. Osland and L. Saks, JHEP 0108 (2001) 050, hep-ph/0105072.

[22] M. Binger and S. J. Brodsky, Phys. Rev. D 74 (2006) 054016, hep-ph/0602199.

[23] L.F. Abbott, Nucl. Phys. B 185 (1981) 189.

[24] L. F. Abbott, M. T. Grisaru and R. K. Schaefer, Nucl. Phys. B 229 (1983) 372.

[25] A. Denner, G. Weiglein and S. Dittmaier, Phys. Lett. B 333 (1994) 420.

[26] J. Papavassiliou, Phys. Rev. D 51 (1995) 856.

[27] A. I. Davydychev, P. Osland and O. V. Tarasov, Phys. Rev. D 58 (1998) 036007, hep-ph/9801380.

[28] A. I. Davydychev and P. Osland, Phys. Rev. D 59 (1999) 014006, hep-ph/9806522.

[29] J. A. Gracey, Phys. Rev. D 84 (2011) 085011, arXiv:1108.4806 [hep-ph].

[30] Z. Bern and D. A. Kosower, Phys. Rev. Lett. 66 (1991) 1669.

[31] Z. Bern and D. A. Kosower, Nucl. Phys. B 362 (1991) 389.

[32] Z. Bern and D. A. Kosower, Nucl. Phys. B 379 (1992) 451.

[33] J. Cornwall, arXiv:1311.1827 [hep-ph], to appear in the proceedings of this workshop.

[34] M. J. Strassler, Nucl. Phys. B 385 (1992) 145, hep-ph/9205205.

[35] M. J. Strassler, "Field theory without Feynman diagrams: a demonstration using actions induced by heavy particles", SLAC-PUB-5978 (1992) (unpublished).

[36] C. Schubert, Eur. Phys. J. C 5 (1998) 693, hep-th/9710067.

[37] C. Schubert, Phys. Rept. 355 (2001) 73, arXiv:hep-th/0101036.

[38] N. Ahmadiniaz, C. Schubert and V.M. Villanueva, JHEP 1301 (2013) 312, arXiv:1211.1821 [hep-th].

[39] N. Ahmadiniaz and C. Schubert, Nucl. Phys. B 869 (2013) 417, arXiv:1210.2331 [hep-ph].

[40] N. Ahmadiniaz and C. Schubert, "String-inspired form factor decompositions of the four-gluon vertex", in preparation.

[41] M. Reuter, M. G. Schmidt and C. Schubert, Ann. Phys. (N.Y.) 259 (1997) 313, hep-th/9610191.

[42] D. Fliegner, P. Haberl, M.G. Schmidt and C. Schubert, Ann. Phys. (N.Y.) 264 (1998) 51, hep-th/9707189.

[43] J. Broedel and L. J. Dixon, JHEP 1210 (2012) 091, arXiv:1208.0876 [hep-th]. 